\title{
Cost-effective on-demand associative author name disambiguation
}

\author{
Adriano Veloso, Anderson A. Ferreira, Marcos André Gonçalves*, Alberto H.F. Laender, \\ Wagner Meira Jr.
}

Departamento de Ciência da Computação, Universidade Federal de Minas Gerais, Brazil

\section{A R T I C L E I N F O}

\section{Article history:}

Received 8 April 2010

Received in revised form 9 August 2011

Accepted 12 August 2011

Available online 1 October 2011

\section{Keywords:}

Machine learning

Digital libraries

Author name disambiguation

Associative methods

Lazy strategies

\begin{abstract}
A B S T R A C T
Authorship disambiguation is an urgent issue that affects the quality of digital library services and for which supervised solutions have been proposed, delivering state-of-the-art effectiveness. However, particular challenges such as the prohibitive cost of labeling vast amounts of examples (there are many ambiguous authors), the huge hypothesis space (there are several features and authors from which many different disambiguation functions may be derived), and the skewed author popularity distribution (few authors are very prolific, while most appear in only few citations), may prevent the full potential of such techniques. In this article, we introduce an associative author name disambiguation approach that identifies authorship by extracting, from training examples, rules associating citation features (e.g., coauthor names, work title, publication venue) to specific authors. As our main contribution we propose three associative author name disambiguators: (1) EAND (Eager Associative Name Disambiguation), our basic method that explores association rules for name disambiguation; (2) LAND (Lazy Associative Name Disambiguation), that extracts rules on a demand-driven basis at disambiguation time, reducing the hypothesis space by focusing on examples that are most suitable for the task; and (3) SLAND (SelfTraining LAND), that extends LAND with self-training capabilities, thus drastically reducing the amount of examples required for building effective disambiguation functions, besides being able to detect novel/unseen authors in the test set. Experiments demonstrate that all our disambigutators are effective and that, in particular, SLAND is able to outperform stateof-the-art supervised disambiguators, providing gains that range from $12 \%$ to more than $400 \%$, being extremely effective and practical.
\end{abstract}

(c) 2011 Elsevier Ltd. All rights reserved.

\section{Introduction}

Citations (here understood as a set of bibliographic features such as author and coauthor names, work title and publication venue title, of a particular publication) are an essential component of many current digital libraries (DLs) and similar systems (Lee, Kang, Mitra, Giles, \& On, 2007). Citation management within DLs involves a number of tasks. One task in particular, name disambiguation, has required significant attention from the research community due to its inherent difficulty. Name ambiguity in the context of bibliographic citations occurs when one author can be correctly referred to by multiple name variations (synonyms) or when multiple authors have exactly the same name or share the same name variation (polysems). This problem may occur for a number of reasons, including the lack of standards and common practices, and the decentralized generation of content (e.g., by means of automatic harvesting).

\footnotetext{
* Corresponding author. Tel.: +55 31 34095860; fax: +55 3134095858 .

E-mail address: mgoncalv@dcc.ufmg.br (M.A. Gonçalves).
} 
The name disambiguation task may be formulated as follows. Let $C=\left\{c_{1}, c_{2}, \ldots, c_{k}\right\}$ be a set of citations. Each citation $c_{i}$ has a list of attributes which includes at least author names, work title and publication venue title. The objective is to produce a disambiguation function which is used to partition the set of citations into $n$ sets $\left\{a_{1}, a_{2}, \ldots, a_{n}\right\}$, so that each partition $a_{i}$ contains (all and ideally only all) the citations in which the $i$ th author appears.

To disambiguate the bibliographic citations of a digital library, first we may split the set of citations into groups of ambiguous authors, called ambiguous groups (i.e., groups of citations having authors with similar names). The ambiguous groups may be obtained, for instance, by using a blocking method (On, Lee, Kang, \& Mitra, 2005). Blocking methods address scalability issues, avoiding the need for comparisons among all citations.

The complexity of dealing with ambiguous names in DLs has led to myriad of methods for name disambiguation (Bhattacharya \& Getoor, 2006, 2007; Culotta, Kanani, Hall, Wick, \& McCallum, 2007; Han, Giles, Zha, Li, \& Tsioutsiouliklis, 2004; Han, Xu, Zha, \& Giles, 2005; Han, Zha, \& Giles, 2005; Huang, Ertekin, \& Giles, 2006; Malin, 2005; On et al., 2005; Song, Huang, Councill, Li, \& Giles, 2007; Torvik \& Smalheiser, 2009; Cota, Ferreira, Nascimento, Gonçalves, \& Laender, 2010). Some of the most effective methods seem to be based on the application of supervised machine learning techniques. In this case, we are given an input data set called the training data (denoted as $\mathcal{D}$ ) which consists of examples, or, more specifically, citations for which the correct authorship is known. Each example is composed of a set of $m$ features $\left(f_{1}, f_{2}, \ldots, f_{m}\right)$ (e.g., corresponding to coauthor names or words in titles) along with a special variable called the author. This author variable draws its value from a discrete set of labels $\left(a_{1}, a_{2}, \ldots, a_{n}\right)$, where each label uniquely identifies an author. The training data is used to produce a disambiguator that relates the features in the training data to the correct author. The test set (denoted as $\mathcal{T}$ ) for the disambiguation problem consists of a set of citations for which the features are known while the correct author is unknown. The disambiguator, which is a function from $\left\{f_{1}, f_{2}, \ldots, f_{m}\right\}$ to $\left\{a_{1}, a_{2}, \ldots, a_{n}\right\}$, is used to predict the correct author of citations in the test set.

Although successful cases have been reported (Han et al., 2004; Han, Xu, et al., 2005; Torvik \& Smalheiser, 2009), some particular challenges associated with author name disambiguation (in the context of bibliographic citations), prevent the full potential of supervised machine learning techniques:

- The acquisition of training examples requires skilled human annotators to manually label citations. Annotators may face hard-to-label citations with highly ambiguous authors. The cost associated with this labeling process thus may render vast amounts of examples unfeasible. The acquisition of unlabeled citations, on the other hand, is relatively inexpensive. However, it may be worthwhile annotating at least some examples, provided that this effort will be then rewarded with an improvement in disambiguation effectiveness. Thus, disambiguators must be cost-effective, achieving high effectiveness even in the case of limited labeling efforts.

- There is a potentially large number of features and authors, and consequently, the number of possible disambiguation functions that may be derived from them is huge. Selecting an appropriate function, given so many possibilities, is challenging. Thus, disambiguators must focus on producing only functions that are suitable to disambiguate specific citations.

- The number of citations in which a particular author is included is extremely skewed. Specifically, few authors are very popular, appearing in several citations, while most of the authors publish only few papers. The effective disambiguation of less popular authors is particularly challenging, since, in such cases, only few examples are available for building a disambiguation function. On the other hand, this is particularly important, because these authors, collectively, may appear in the majority of the citations. Thus, disambiguators must exploit all available evidence, even if such evidence is associated with rarely appearing authors.

- It is not reasonable to assume that all possible authors are included in the training data (specially due to the scarce availability of examples). Thus, disambiguators must be able to detect unseen/unknown authors, for whom no label was previously specified.

There are countless strategies for devising a name disambiguator for bibliographic citations. One of these strategies is to exploit dependencies and associations between bibliographic features and authors. These associations are usually hidden in the examples and, when uncovered, they may reveal important aspects concerning the underlying characteristics of each author (i.e., typical coauthors, typical publication venues, writing patterns, and any combination of these aspects). Obviously, these aspects are evidence that may be exploited for the sake of predicting the correct author of a citation. This is the strategy adopted by associative disambiguators, where the disambiguation function is built from rules of the form $\mathcal{X} \rightarrow a_{i}$ (where $\mathcal{X}$ is a set of features and $a_{i}$ is an author label). Exploiting associations hidden in examples has shown to be valuable in many applications, including ranking (Veloso, Mosrri, \& Gonçalves, 2008), and document categorization (Veloso, Cristo, Gonçalves, \& Zaki, 2006).

In this article, we introduce an associative author name approach that identifies authorship by extracting, from training examples, rules associating citation features (e.g., coauthor names, work title, publication venue) to specific authors. In particular, we propose three distinct associative disambiguators: EAND (Eager Associative Name Disambiguator), LAND (Lazy Associative Name Disambiguator), and SLAND (Self-Training LAND). The proposed disambiguators apply a probabilistic strategy that continuously exploits evidence in favor or against each author in an ambiguous group. Our main focus is on the effectiveness of the name disambiguation process, i.e., in assigning the correct author to the citation as frequently as possible, but we also show that our lazy solutions are efficient, i.e., they are polinomial in the number of exploited features. The contributions of this article are highlighted by specific properties of these disambiguators: 
- EAND, which works in a eager manner, provides the basic foundations for the use of association rules for name disambiguation.

- LAND extracts rules from the examples on a demand-driven basis, according to the citation being disambiguated. Thus, instead of producing a single disambiguation function that is good on average (considering all citations in the test set), LAND follows a lazy strategy that delays the inductive process until a citation is given for disambiguation. Then, a specific disambiguation function is produced for that citation. This citation-centric strategy ensures that evidence coming from citations belonging to less popular authors are not neglected during rule extraction. Thus, this strategy is specially well suited for ambiguous groups where the popularity distribution of authors is skewed. Further, extracting rules on a demand-driven basis also reduces the hypothesis space, since there is a concentration on extracting only rules that are relevant to the specific citation being considered.

- To limit labeling efforts (which is a major problem in real world scenarios), SLAND extends LAND by employing a selftraining strategy, in which a reliable prediction is considered as a new example and is included in the training data. Since rules are extracted on a demand-driven basis, at disambiguation time, the next citation to be processed will possibly take advantage of the recently included (pseudo-)example.

- SLAND uses the lack of enough evidence (i.e., rules) supporting any known author present in the training data, to detect the appearance of a novel/unseen author in the test set. In such case, a new label is associated with this novel author, and the corresponding citation is considered as a new example which is included in the training data.

The proposed associative disambiguators are intuitive (easily understood using a set of illustrative examples), and also extremely effective, as it will be shown by a systematic set of experiments using citations extracted from the DBLP ${ }^{1}$ and BDBComp $^{2}$ collections. The results show that, while EAND is in close rivalry with previously representative supervised disambiguators, LAND is able to outperform all of them with gains in terms of macroF f $_{1}$ of more than $12 \%$. Improvements reported by SLAND are also impressive, showing the advantages of its self-training ability specially when there is a scarce availability of examples.

The rest of this article is organized as follows. Section 2 discusses related work. Section 3 introduces our associative name disambiguation approach and the three new disambiguators and their properties in details. Section 4 presents our experimental evaluation. Finally, Section 5 concludes the article with some discussion about future work.

\section{Related work}

Existing name disambiguation methods adopt a wide spectrum of solutions that range from those based on supervised learning techniques (Han et al., 2004) to those that use some unsupervised or semi-supervised strategy (Bhattacharya \& Getoor, 2006, 2007; Culotta et al., 2007; Han, Xu, et al., 2005; Han, Zha, \& Giles, 2005; Huang et al., 2006; On et al., 2005; Song et al., 2007; Torvik, Weeber, Swanson, \& Smalheiser, 2005) or follow a graph-oriented approach (Malin, 2005; On \& Lee, 2007; On, Elmacioglu, Lee, Kang, \& Pei, 2006). In this section, we present a brief review of some representative author name disambiguation methods. Our main focus, however, is on those methods that have been specifically designed for addressing the author name disambiguation problem in the context of bibliographic citations, since they are more related to the scope of our work.

Being some of the first to address the problem, Han et al. (2004) propose two methods based on supervised learning techniques that use coauthor names, work titles and publication venue titles as evidence for name disambiguation. The first method uses a naive Bayes model to capture all writing patterns in the authors' citations whereas the second method is based on Support Vector Machines (SVMs). Both methods have been evaluated using two collections, one from the Web (mainly publication lists from homepages), and the other from DBLP.

In Han, Zha, et al. (2005), the authors propose an unsupervised method for name disambiguation that uses k-way spectral clustering. This method was also evaluated with collections extracted from the Web and from DBLP. The results showed that this method has achieved 63\% of accuracy in the collection extracted from DBLP, and $71.2 \%$, and $84.3 \%$ of accuracy in the collections extracted from the Web.

In Torvik et al. (2005), the authors propose a probabilistic metric for determining the similarity between MEDLINE records. The learning model is created using similarity profiles between articles. A similarity profile is a comparison vector between a pair of articles, used to indicate the similarity between them, based on the following attributes: work title, publication venue title, coauthor and author names, medical subject headings, language, and affiliation. The authors also propose some heuristics for generating training sets (positive and negative) automatically. When the probabilistic metric receives two records, their similarity profile is computed and the relative frequency of this profile in the positive and negative training sets is checked for determining whether these two records are authored by the same author or not. In Torvik and Smalheiser (2009), the authors extend their method including the addition of new features, new ways of automatically generating training sets, an improved algorithm for correcting the transitivity problem, and a new agglomerative clustering algorithm.

\footnotetext{
1 http://dblp.uni-trier.de.

2 http://www.lbd.ufmg.br/bdbcomp.
} 
On et al. (2005) present a comparative study of disambiguation strategies based on a two-step framework. In the first step (blocking), similar names are blocked together in order to reduce the number of candidates for the second step (disambiguation), which uses coauthor information to measure the distance between two names in the citations.

On et al. (2006) then propose a graph-based method for disambiguation that uses a graph in which each vertex represents an author and each edge represents a coauthorship between two authors. After ambiguous groups are determined, the method finds the common quasi-clique between two vertices and the vertex degree of this quasi-clique is used as a similarity measure for the respective authors. Results expressed in terms of average ranked precision measures, considering real and synthetic collections, have shown that this method considerably improves the effectiveness of traditional text similarity measures.

Huang et al. (2006) present a framework for name disambiguation in which a blocking method first creates candidate classes of authors with similar names and then DBSCAN, a density-based clustering method (Ester, Kriegel, Sander, \& $\mathrm{Xu}, 1996)$, is used to cluster citations by author. For each block, the distance metric between citations used in DBSCAN is calculated by an online active selection SVM, which yields, according to the authors, a simpler model than those obtained by standard SVMs. This method exploits additional sources of evidence, such as information extracted from the headers of papers corresponding to the respective citations obtained from CiteSeer.

In Bhattacharya and Getoor (2006), the authors extend the Latent Dirichlet Allocation model and propose a probabilistic model for collective entity resolution that uses the cooccurrence of the references to entities ${ }^{3}$ in each work to determine the entities jointly, i.e., they use the disambiguated references to disambiguate other references in the same work. An algorithm for collective entity resolution that uses the attributes and relational information of the citation records is proposed in (Bhattacharya \& Getoor, 2007).

Culotta et al. (2007) propose a more generic representation for the author disambiguation problem that considers features over sets of records, instead of only features between pairs of records, and present a training algorithm that is error-driven, i.e., training examples are generated from incorrect predictions in the training data, and rank-based, i.e., the classifier provides a ranked result for the disambiguation. Whereas in (Kanani, McCallum, \& Pal, 2007), the authors present several methods for increasing the author coreference by gathering additional evidence from the Web.

Cota, Gonçalves, and Laender $(2007,2010)$ propose a heuristic-based hierarchical clustering method for name disambiguation that involves two steps. In the first step, the method creates clusters of citations with similar author names. Then, in the second step, the method successively fuses clusters of citations with similar author names based on several heuristics. In each fusion, the information of fused clusters is aggregated, providing more information for the next round of fusion. This process is successively repeated until no more fusions are possible.

On and Lee (2007) have studied the scalability issue of the disambiguation problem. They examine two state-of-the-art solutions, k-way spectral clustering (Han, Zha, et al., 2005) and multi-way distributional clustering (Bekkerman \& McCallum, 2005), and pointed out their limitations with respect to scalability. Then, using collections extracted from the ACM DL and from DBLP, they showed that a method based on the multi-level graph partition technique (Dhillon, Guan, \& Kulis, 2005) may be successfully applied to name disambiguation in large collections.

In Song et al. (2007), the authors propose a two-step unsupervised method. The first step, after learning the probability distribution of the title and publication venue words and author names, uses Probabilistic Latent Semantic Analysis and Latent Dirichlet Allocation to assign a vector of probabilities of topics to a name. In the second step, they consider the probability distribution of topics with respect to person names as new evidence for name disambiguation.

Kang et al. (2009) explore the use of coauthorship using a Web-based technique that obtains implicit coauthors of the author to be disambiguated. They submit as query a pair of citation author names to Web search engines to retrieval documents that contains both author names, and to extract the new names found in these documents as new implicit coauthors of the pair.

Pereira et al. (2009) also exploit the Web for obtaining additional information to disambiguate author names. The proposed method attempts to find Web documents corresponding to curricula vitae or Web pages containing publications of a single author. If two citations of two ambiguous authors occur in the same Web document, these citations are considered as belonging to the same author and are fused in a same cluster. One problem with this method and the previous one is the additional cost of extracting all the needed information from Web documents.

Finally, Treeratpituk and Giles (2009) propose a pairwise linkage function for author name disambiguation in the Medline digital library. The authors exploit a large feature set obtained from Medline metadata, similar to that of (Torvik et al., 2005), and assess the effectiveness of random forests, in comparison to other classifiers, for constructing a pairwise linkage function to be used in some author name disambiguation algorithms. They also investigate subsets of the features capable of reaching good effectiveness.

Since name disambiguation is not restricted to a single context, it is worth noting that several other disambiguation methods, which exploit distinct sources of evidence or are targeted to other applications. For instance, Malin (2005) propose two methods for name disambiguation that exploit the existing relations among ambiguous names. The first method is based on a hierarchical clustering strategy and the second one makes use of social networks. Vu, Masada, Takasu, and Adachi (2007) propose the use of Web directories as a knowledge base to disambiguate personal names in Web search results,

\footnotetext{
${ }^{3}$ Here each author name in a work corresponds to a reference to an author.
} 
whereas Bekkerman and McCallum (2005) present two methods for addressing this same problem, one based on the link structure of Web pages, the other one using agglomerative/conglomerative double clustering, a multi-way distributional clustering. A deeper discussion of these methods, however, are out of the scope of this paper.

Despite all such efforts, problems due to the huge hypothesis space and the skewed popularity distribution of authors are often neglected. Further, another difficulty is imposed by practical constraints, which may render vast amounts of examples unfeasible. All these problems may prevent the full potential of supervised disambiguation methods. Thus, addressing these problems is an opportunity for improvement, and is the target of our study.

\section{Associative disambiguation}

Associative name disambiguation, in the context of bibliographic citations, exploits the fact that, frequently, there are strong associations between bibliographic features $\left(f_{1}, f_{2}, \ldots, f_{m}\right)$ and specific authors $\left(a_{1}, a_{2}, \ldots, a_{n}\right)$. Here, we consider as feature each coauthor name and each word in work or publication venue titles. The learning strategy adopted by associative disambiguators is based on uncovering such associations from the training data, and then building a function $\left\{f_{1}, f_{2}, \ldots, f_{m}\right\} \rightarrow\left\{a_{1}, a_{2}, \ldots, a_{n}\right\}$ using such associations. Typically, these associations are expressed using rules of the form $\mathcal{X} \rightarrow a_{1}, \mathcal{X} \rightarrow a_{2}, \ldots, \mathcal{X} \rightarrow a_{n}$, where $\mathcal{X} \subseteq\left\{f_{1}, f_{2}, \ldots, f_{m}\right\}$. For example, $\{$ coauthor $=K$. Talwar, title $=$ Metric, venue $=$ LATIN $\} \rightarrow a_{1}$ while $\{$ coauthor $=W$. Lin, title $=$ Optimal, title $=$ Sparse $\} \rightarrow a_{2}$ are two association rules indicating that the coauthor name "K. Talkar", the word "Metric" in the work title and "LATIN" in the publication venue title are associated with the author $a_{1}$ (Anupam Gupta) and the coauthor name "W. Lin" and the words "Optimal" and "Sparse" in the work title are associated with the author $a_{2}$ (Chuen-Liang Chen).

In the following discussion we will denote as $\mathcal{R}$ an arbitrary rule set. Similarly, we will denote as $\mathcal{R}_{a_{i}}$ a subset of $\mathcal{R}$ which is composed of rules of the form $\mathcal{X} \rightarrow a_{i}$ (i.e., rules predicting author $a_{i}$ ). A rule $\mathcal{X} \rightarrow a_{i}$ is said to match citation $x$ if $\mathcal{X} \subseteq x$ (i.e., $x$ contains all features in $\mathcal{X}$ ), and these rules form the rule set $\mathcal{R}_{a_{i}}^{x}$. That is, $\mathcal{R}_{a_{i}}^{x}$ is composed of rules predicting author $a_{i}$ and matching citation $x$. Obviously, $\mathcal{R}_{a_{i}}^{x} \subseteq \mathcal{R}_{a_{i}} \subseteq \mathcal{R}$.

Naturally, there is a total ordering among rules, in the sense that some rules show stronger associations than others. A widely used statistic, called confidence (Agrawal, Imielinski, \& Swami, 1993) (denoted as $\theta\left(\mathcal{X} \rightarrow a_{i}\right)$ ), measures the strength of the association between $\mathcal{X}$ and $a_{i}$. The confidence of the rule $\mathcal{X} \rightarrow a_{i}$ is simply calculated by the conditional probability of $a_{i}$ being the author of citation $x$, given that $\mathcal{X} \subseteq \mathcal{X}$.

Using a single rule to predict the correct author may be prone to error. Instead, the probability (or likelihood) of $a_{i}$ being an author of citation $x$ is estimated by combining rules in $\mathcal{R}_{a_{i}}^{x}$. More specifically, $\mathcal{R}_{a_{i}}^{x}$ is interpreted as a poll, in which each rule $\mathcal{X} \rightarrow a_{i} \in \mathcal{R}_{a_{i}}^{x}$ is a vote given by features in $\mathcal{X}$ for author $a_{i}$. The weight of a vote $\mathcal{X} \rightarrow a_{i}$ depends on the strength of the association between $\mathcal{X}$ and $a_{i}$, which is $\theta\left(\mathcal{X} \rightarrow a_{i}\right)$. The process of estimating the probability of $a_{i}$ being the author of citation $x$ starts by summing weighted votes for $a_{i}$ and then averaging the obtained value by the total number of votes for $a_{i}$, as expressed by the score function $s\left(a_{i}, x\right)$ shown in Eq. (1) (where $r_{j} \subseteq \mathcal{R}_{a_{i}}^{x}$ and $\left|\mathcal{R}_{a_{i}}^{x}\right|$ is the number of rules in $\left.\mathcal{R}_{a_{i}}^{x}\right)$. Thus, $s\left(a_{i}, x\right)$ gives the average confidence of the rules in $\mathcal{R}_{a_{i}}^{x}$ (obviously, the higher the confidence, the stronger the evidence of authorship).

$$
s\left(a_{i}, x\right)=\frac{\sum_{j=1}^{\left|\mathcal{R}_{i_{i}}^{x}\right|} \theta\left(r_{j}\right)}{\left|\mathcal{R}_{a_{i}}^{x}\right|}
$$

The estimated probability of $a_{i}$ being an author of citation $x$, denoted as $\hat{p}\left(a_{i} \mid x\right)$, is simply obtained by normalizing $s\left(a_{i}, x\right)$, as shown in Eq. (2). A higher value of $\hat{p}\left(a_{i} \mid x\right)$ indicates a higher likelihood of $a_{i}$ being an author of $x$. The author associated with the highest likelihood is finally predicted as the correct author of citation $x$.

$$
\hat{p}\left(a_{i} \mid x\right)=\frac{s\left(a_{i}, x\right)}{\sum_{j=1}^{n} s\left(a_{j}, x\right)}
$$

Next, we will introduce novel associative name disambiguators: EAND, LAND, and SLAND. We will start by discussing EAND, since it is the simplest disambiguator. Then, we will discuss LAND, which employs a more sophisticated rule extraction strategy. Lastly, we will SLAND, which extends LAND, being less sensitive to scarce training and the presence of new authors.

\subsection{Eager Associative Name Disambiguation}

Rule extraction is a major issue when devising an associative disambiguator. Extracting all rules from the training data is infeasible and, thus, pruning strategies are employed in order to reduce the number of rules that are processed. A simple pruning strategy is based on a support threshold, $\sigma_{\min }$, which separates frequent from infrequent rules. This is the strategy adopted by EAND, the Eager Associative Name Disambiguator that will be presented in this section.

The $\sigma_{\min }$ threshold produces a minimum cut-off value, $\pi_{\min }$, as shown in Eq. (3) (where ceil( $(z)$ is the nearest integer greater than or equal to $z$ ).

$$
\pi_{\min }=\operatorname{ceil}\left(\sigma_{\min } \times|\mathcal{D}|\right)
$$


Table 1

Illustrative example (ambiguous group of A. Gupta).

\begin{tabular}{|c|c|c|c|c|}
\hline & Label & Coauthors & Publication title & Venue \\
\hline$c_{1}$ & $a_{1}$ & K. Talwar & How to Complete a Doubling Metric & LATIN \\
\hline$c_{2}$ & $a_{1}$ & T. Chan, K. Talwar & Ultra-Low-Dimensional Embeddings for Doubling Metrics & SODA \\
\hline$c_{3}$ & $a_{1}$ & T. Chan & Approximating TSP on Metrics with Global Growth & SODA \\
\hline$c_{4}$ & $a_{1}$ & T. Chan (among others) & Metric Embeddings with Relaxed Guarantees & FOCS \\
\hline$c_{5}$ & $a_{2}$ & T. Ashwin, S. Ghosal & Adaptable Similarity Search using Non-Relevant Information & VLDB \\
\hline$c_{6}$ & $a_{2}$ & - & Explanation-Based Failure Recovery & AAAI \\
\hline$c_{7}$ & $a_{2}$ & M. Bhide (among others) & Dynamic Access Control Framework Based on Events & ICDE \\
\hline$c_{8}$ & $a_{3}$ & S. Sarawagi & Creating Probabilistic DBs from Information Extraction Models & VLDB \\
\hline$c_{9}$ & $a_{3}$ & S. Puradkar (among others) & Semantic Web Based Pervasive Computing Framework & AAAI \\
\hline$c_{10}$ & $a_{4}$ & V. Harinarayan, A. Rajaraman & Virtual Database Technology & ICDE \\
\hline$c_{11}$ & $\left(a_{1}\right) ?$ & K. Talwar & Approximating Unique Games & SODA \\
\hline$c_{12}$ & $\left(a_{4}\right) ?$ & V. Harinarayan & Index Selection for OLAP & ICDE \\
\hline$c_{13}$ & $\left(a_{4}\right) ?$ & I. Mumick & What is the DW Problem? & VLDB \\
\hline$c_{14}$ & $\left(a_{4}\right) ?$ & V. Harinarayan & Aggregate-Query Processing in DW Environments & VLDB \\
\hline$c_{15}$ & $\left(a_{5}\right) ?$ & J. Hennessy (among others) & Flexible Use of Memory in DSM Multi-processors & ISCA \\
\hline$c_{16}$ & $\left(a_{5}\right) ?$ & J. Hennessy (among others) & Impact of Flexibility in the FLASH Multi-processors & ASPLOS \\
\hline
\end{tabular}

The number of citations in the training data in which rule $\mathcal{X} \rightarrow a_{i}$ has occurred is denoted as $\pi\left(\mathcal{X} \rightarrow a_{i}\right)$ and this rule is frequent if it is supported by at least $\pi_{\min }$ citations in the training data (i.e., $\left.\pi\left(\mathcal{X} \rightarrow a_{i}\right) \geqslant \pi_{\min }\right)$. Ideally, infrequent rules are not important. However, most of the authors appear in very few citations and, thus, rules predicting such authors are very likely to be infrequent and, consequently, they are not included in $\mathcal{R}$. These infrequent feature-author associations may be important for the sake of disambiguation and, therefore, disambiguation effectiveness is seriously harmed when such rules are pruned.

Algorithm 1. Eager Associative Name Disambiguation.

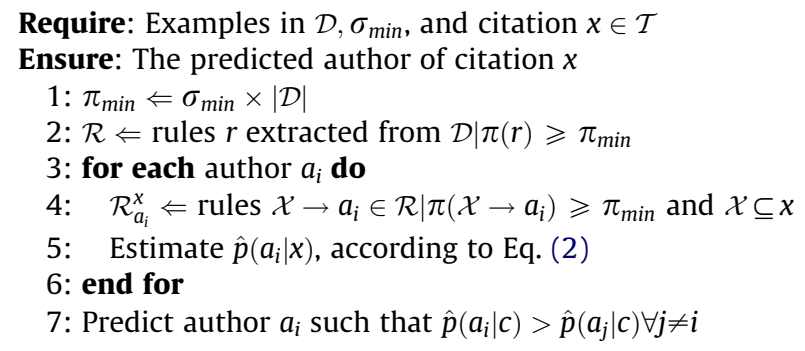

A naive solution is to lower the value of $\sigma_{\min }$, so that rules predicting less popular authors are also included in $\mathcal{R}$. This solution, however, may be disastrous as the amount of rules that are processed may increase in a very large pace (a problem known as rule explosion). Even worse, most of these rules are useless for the sake of disambiguation. ${ }^{4}$ An optimal value of $\sigma_{\min }$ is unlikely to exist, and tuning is generally driven by intuition and prone to error as a consequence. The main steps of EAND are shown in Algorithm 1. There is a vast amount of association rule mining algorithms (Goethals \& Zaki, 2004) and we assume that any of these algorithms can be used (or modified) to enumerate the rules from the training data.

Example. Consider the citations shown in Table 1. These citations were collected from DBLP and are used as a running example in this paper. Each citation contains author names, words in publication and venue titles. In the training data, there are four different authors with the same name - "A. Gupta" (i.e., $a_{1}, a_{2}, a_{3}$, and $a_{4}$ ). Author $a_{1}$ appears in four citations and is the most popular/prolific one, while author $a_{4}$ appears in only one citation and is the least popular/prolific one (in the training data). There are six citations in the test set (i.e., the last six citations).

Suppose we set $\sigma_{\min }=0.20$. In this case, according to Eq. (3), $\pi_{\min }=2$ (since $|\mathcal{D}|=10$ ). For this cut-off value, more than 50 rules are included in $\mathcal{R}$. One of these rules is:

$$
\text { coauthor }=\mathrm{K} \text {. Talwar } \wedge \text { venue }=\operatorname{SODA} \stackrel{\theta=1.00}{\longrightarrow} a_{1} \text {. }
$$

\footnotetext{
${ }^{4}$ A rule is useless if it does not match any citation in $\mathcal{T}$.
} 
Suppose we want to predict the correct author of citation $c_{11}$ using $\mathcal{R}$. The first step is to filter only rules matching $c_{11}$, forming the rule set $\mathcal{R}^{c_{11}}$. All rules in $\mathcal{R}^{c_{11}}$ predict the same author, $a_{1}$ (i.e., $\left|\mathcal{R}_{a_{1}}\right|=\left|\mathcal{R}_{a_{1}}^{c_{11}}\right|$ ). In this case, the estimated probability of $a_{1}$ being the author of citation $c_{11}$ is $\hat{p}\left(a_{1} \mid c_{11}\right)=1.00$ (i.e., all the rules in $\mathcal{R}^{c_{11}}$ predict the most prolific author, $\left.a_{1}\right)$, and, thus, $a_{1}$ is the predicted author. In fact, $a_{1}$ is the correct author of citation $c_{11}$. Now, suppose we want to predict the correct author of citation $c_{12}$. In this case, for $\sigma_{\min }=0.20$, there is no rule in $\mathcal{R}^{c_{12}}$. The typical strategy of predicting the most popular author would predict again author $a_{1}$. However, $a_{4}$ turns to be the correct author of citation $c_{12}$. The wrong prediction has occurred because rules predicting author $a_{4}$ are not frequent enough for $\sigma_{\min }=0.20$. If we drop $\sigma_{\min }$ to 0.10 , then a very large number of rules is extracted from the training data and most of these rules are useless for predicting the author of citation $c_{12}$ (incurring unnecessary, sometimes prohibitive, overhead). in fact, this preference for more popular authors is very problematic for the author name disambiguation task, because most of the authors appear in only few citations. In the following, we propose a strategy that addresses this problem.

\subsection{Lazy Associative Name Disambiguation}

An ideal disambiguator would extract only useful rules from $\mathcal{D}$, without discarding important ones. Citations in the test set have valuable information that may be used during rule extraction to guide the search for useful and important rules. LAND, the Lazy Associative Name Disambiguator to be presented in this section, exploits such information.

\subsubsection{On-demand rule generation}

We propose to extract only useful rules, while reducing the chance of discarding important ones, by extracting rules on a demand-driven basis, according to the citation being considered. Specifically, whenever a citation $x \in \mathcal{T}$ is being considered, that citation is used as a filter to remove irrelevant features (and often entire examples) from $\mathcal{D}$, forming a projected training data, $\mathcal{D}^{x}$, which contains only features that are included in citation $x$ (Veloso et al., 2006). This process reduces the size and dimensionality of the training data (and consequently, it also reduces the hypothesis space), focusing only on features and examples that are most suitable to disambiguate a specific citation.

Algorithm 2. Lazy Associative Name Disambiguation.

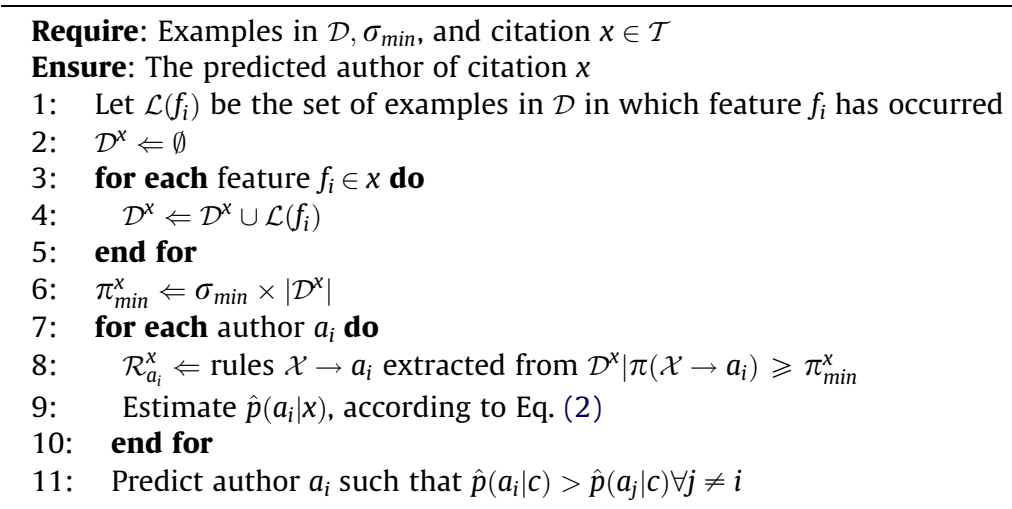

\subsubsection{Pruning with multiple cut-off values}

A typical strategy used to prevent support-based over-pruning (i.e., discarding important rules) is to use a different cutoff value, which is a function of the popularity of authors. More specifically, the cut-off value is higher for rules predicting more popular authors, and lower for rules predicting less popular ones. The problem with this strategy is that it does not take into account the frequency of the features composing the rule and, thus, if an important rule is composed of rare features, it will be discarded, specially if this rule predicts a very popular author. We propose an alternate strategy that employs multiple cut-off values, which are calculated depending on the frequency of the features composing a citation. Intuitively, if a citation $x \in \mathcal{T}$ contains frequent features (i.e., these features occur in many citations in $\mathcal{D}$ ), then the size of the projected training data will be large. Otherwise, if a citation $x$ contains rare features (i.e., these features occur only in few citations in $\mathcal{D}$ ), then the size of the projected training data will be small. For a fixed value of $\sigma_{\min }$, the cut-off value for a specific citation $x$, denoted as $\pi_{\min }^{x}$, is calculated based on the size of the corresponding projected training data, as shown in Eq. (4).

$$
\pi_{\min }^{x}=\operatorname{ceil}\left(\sigma_{\min } \times\left|\mathcal{D}^{x}\right|\right)
$$

The cut-off value applied while considering citation $x$ varies from $1 \leqslant \pi_{\min }^{x} \leqslant \sigma_{\min } \times|\mathcal{D}|$, which is bounded by $\sigma_{\min } \times|\mathcal{D}|$ (the single cut-off value applied by EAND). Therefore, the chance of discarding important (but less frequent) rules is reduced. The main steps of LAND are shown in Algorithm 2. 
Table 2

$\mathcal{D}^{c_{12}}$, Training data projected for $c_{12}$.

\begin{tabular}{llll}
\hline & Label & Coauthors & Publication title \\
\hline$c_{7}$ & $a_{2}$ & - & - \\
$c_{10}$ & $a_{4}$ & V. Harinarayan & ICDE \\
\hline
\end{tabular}

\subsubsection{Computation complexity}

LAND efficiently extracts rules from the training data. It is demonstrated in the Theorem 1 :

Theorem 1. The complexity of LAND increases polynomially with the number of features in the collection.

Proof. Let $n$ be the number of features in the collection. Obviously, the number of possible association rules that can be extracted from $\mathcal{D}$ is $2^{n}$. Also, let $x$ be an arbitrary citation in $\mathcal{T}$. Since It contains at most $k$ features (with $k \ll n$ ), any rule useful for predicting the author of the citation $x$ can have at most $k$ features in its antecedent. Therefore, the number of possible rules that are useful for predicting the author of the citation $x$ is $(n-k) \times\left(k+\left(\begin{array}{l}k \\ 2\end{array}\right)+\cdots+\left(\begin{array}{l}k \\ k\end{array}\right)\right)=O\left(n^{k}\right)($ since $k \ll n)$,
and thus, the number of useful rules increases polynomially in $n . \quad \square$

Example. Suppose again that we want to predict the author of citation $c_{12}$. The value of $\sigma_{\min }$ is, again, set to 0.20 . The projected training data for $c_{12}, \mathcal{D}^{c_{12}}$, as shown in Table 2, contains only two citations, $c_{7}$ and $c_{10}$ (note that $\mathcal{L}($ venue $=\mathrm{ICDE})=\left\{c_{7}, c_{10}\right\}$ and $\mathcal{L}(\mathrm{co}-$ author $=\mathrm{V}$. Harinarayan $\left.)=\left\{c_{10}\right\}\right)$. Thus, $\pi_{\min }^{c_{12}}=1$, but even for such low cut-off value, only four rules can be extracted from $\mathcal{D}^{c_{12}}$ (since irrelevant features were removed), which are:

$$
\begin{aligned}
& \text { coauthor }=\text { V. Haribarayan } \stackrel{\theta=1.00}{\rightarrow} a_{4} \\
& \text { coauthor }=\text { V. Haribarayan } \wedge \text { venue }=\operatorname{ICDE} \stackrel{\theta=1.00}{\rightarrow} a_{4} \\
& \text { venue }=\operatorname{ICDE} \stackrel{\theta=0.50}{\rightarrow} a_{4} \\
& \text { venue }=\operatorname{ICDE} \stackrel{\theta=0.50}{\rightarrow} a_{2}
\end{aligned}
$$

According to Eq. (1), $s\left(a_{2}, c_{12}\right)=0.50$ and $s\left(a_{4}, c_{12}\right)=0.83$. The estimated probability of $a_{2}$ being the author of citation $c_{12}$, according to Eq. (2), is $\hat{p}\left(a_{2} \mid c_{12}\right)=\frac{0.50}{0.50+0.83}=0.38$, while $\hat{p}\left(a_{4} \mid c_{12}\right)=\frac{0.83}{0.50+0.83}=0.62$. Therefore, $a_{4}$ is correctly predicted as the author of citation $c_{12}$. Although simple, this example allows us to grasp that the ability to extract rules on a demand-driven basis, by projecting the training data according to specific citations, makes LAND well-suited to find authors that appear in only few citations.

An interesting problem occurs when we consider citation $c_{13}$. In this case, after extracting the rules from $\mathcal{D}^{c_{13}}$ and then applying Eqs. (1) and (2), we finally obtain $\hat{p}\left(a_{2} \mid c_{13}\right)=0.50$ and $\hat{p}\left(a_{3} \mid c_{13}\right)=0.50$. Both predictions, $a_{2}$ and $a_{3}$, are equally likely to be correct, and more training examples are needed in order to perform a more reliable prediction.

Another interesting problem occurs when we consider citation $c_{15}$. In this case, it turns out that, after projecting the training data according to $c_{15}$, there is no remaining example (i.e., $\mathcal{D}^{c_{15}}=\emptyset$ ). This means that there is no rule in the training data supporting any known author for $c_{15}$, and, thus no reliable prediction can be performed. Next, we propose a strategy to address these two problems.

\subsection{Self-Training Lazy Associative Name Disambiguation}

In this section we propose SLAND, a Self-training Lazy Associative Name Disambiguator that is able to incorporate new examples to the training data and detect unseen authors that are not present in the original training data. SLAND extends LAND, therefore incorporating its abilities, while solving specific issues that arise in real world scenarios, such as the scarcity of training data and the appearance of unseen ambiguous authors.

\subsubsection{Inclusion of additional examples}

Additional examples may be obtained from the predictions performed by the disambiguator. In this case, reliable predictions are regarded as correct ones, and thus, they can be safely included in the training data. Next we define the reliability of a prediction.

Given an arbitrary citation $c$ in the test set, and the most likely authors for $c, a_{i}$, we denote as $\Delta(c)$ the reliability of predicting $a_{i}$, as shown in Eq. (5).

$$
\Delta(c)=\frac{\hat{p}\left(a_{i} \mid c\right)}{\sum_{j=1}^{n} \hat{p}\left(a_{j} \mid c\right)}
$$


The idea is to only predict $a_{i}$ if $\Delta(c) \geqslant \Delta_{\min }$, where $\Delta_{\min }$ is a user specified parameter which indicates the minimum reliability necessary to regard the corresponding prediction as correct, and, therefore, to include it in the training data.

\subsubsection{Temporary abstention}

Naturally, some predictions are not enough reliable for certain values of $\Delta_{\min }$. An alternative is to abstain from such doubtful predictions. As new examples are included in the training data (i.e., the reliable predictions), novel evidence may be exploited, hopefully increasing the reliability of the predictions that were previously abstained. To optimize the usage of reliable predictions, we place citations in a priority queue, so that citations associated with reliable predictions are considered first. The process works as follows. Initially, citations in the test set are randomly placed in the queue. If the author of the citation that is located in the beginning of the queue may be reliably predicted, then the prediction is performed, the citation is removed from the queue and included in the training data as a new example. Otherwise, if the prediction is not reliable, the corresponding citation is simply placed in the end of the queue and will be only processed after all other citations are processed. The process continues performing more reliable predictions first, until no more reliable predictions are possible. The remaining citations (for which only doubtful predictions are possible) are then processed normally, but the corresponding predictions are not included in the training data. The process stops after all citations are processed.

\subsubsection{Detection of unseen authors}

We propose to use the lack of rules supporting any seen author (i.e., authors that are present in the original training data) as an evidence indicating the appearance of an unseen author. The number of rules that is necessary to consider an author as an already seen one is controlled by another user-specified parameter, $\gamma_{\text {min }}$. Specifically, for a citation $c$, if the number of rules extracted from $\mathcal{D}^{c}$ (which is denoted as $\gamma(c)$ ), is smaller than $\gamma_{\min }$ (i.e., $\gamma(c)<\gamma_{\min }$ ), then the author of citation $c$ is considered as a novel/unseen author and a new label $a_{k}$ is created to identify such author. Further, this prediction is considered as a new example and included in the training data. The main steps of SLAND are shown in Algorithm 3.

Example. Suppose again that we want to predict the author of citation $c_{13}$. The value of $\Delta_{\min }$ is set to 1.50 and the value of $\sigma_{\min }$ is, again, set to 0.20 . As discussed in the previous section, $\hat{p}\left(a_{2} \mid c_{13}\right)=\hat{p}\left(a_{3} \mid c_{13}\right)=0.50$ and, therefore, $\Delta\left(c_{13}\right)=\frac{\hat{p}\left(a_{2} \mid c_{13}\right)}{\hat{p}\left(a_{3} \mid c_{13}\right)}=\frac{\hat{p}\left(a_{3} \mid c_{13}\right)}{\hat{p}\left(a_{2} \mid c_{13}\right)}=1.00<\Delta_{\text {min }}$. Thus, the prediction is abstained due to its low reliability and $c_{13}$ is placed in the end of the queue. For the next citation in the queue, $c_{14}$, we have $\hat{p}\left(a_{4} \mid c_{14}\right)=0.75$ and $\hat{p}\left(a_{3} \mid c_{14}\right)=0.50$. In this case, $\Delta\left(c_{14}\right)=\frac{\hat{p}\left(a_{4} \mid c_{14}\right)}{\hat{p}\left(a_{3} \mid c_{14}\right)}=1.50 \geqslant \Delta_{\min }$ and, therefore, predicting $a_{4}$ is considered reliable. Further, citation $c_{14}$ is included in the training data as a new example.

Now, consider the next citation in the queue, $c_{15}$. Also, suppose we set $\gamma_{\min }$ to 1 . No rule can be extracted from $\mathcal{D}^{c_{15}}$ (i.e., $0<\gamma_{\min }$ ) and, thus, the appearance of an unseen author is detected. A new label, $a_{5}$, is associated with this author and $c_{15}$ is included in the training data. The next citation to be processed is $c_{16}$. After including citation $c_{15}$ as a new example, a new rule matching citation $c_{16}$ is extracted from $\mathcal{D}^{c_{16}}$ (i.e., coauthor $=\mathrm{J}$. Hennessy $\stackrel{\theta=1.00}{\rightarrow} a_{5}$ ), and thus we have $\hat{p}\left(a_{5} \mid c_{16}\right)=1.00$, and therefore author $a_{5}$ is the predicted one. Now, there is only one remaining citation to be processed, $c_{13}$, which was previously abstained. After the inclusion of citation $c_{14}$ as a new example, we have $\hat{p}\left(a_{4} \mid c_{13}\right)=0.73$ and $\hat{p}\left(a_{2} \mid c_{13}\right)=\hat{p}\left(a_{3} \mid c_{13}\right)=0.33$. Consequently, $\Delta\left(c_{13}\right)=2.33>\Delta_{\min }$ and $a_{4}$ is considered as a reliable prediction. There is no more citations to be processed, and the process finally stops. In the next section we will evaluate the effectiveness of the proposed disambiguators.

Algorithm 3. Self-Training LAND.

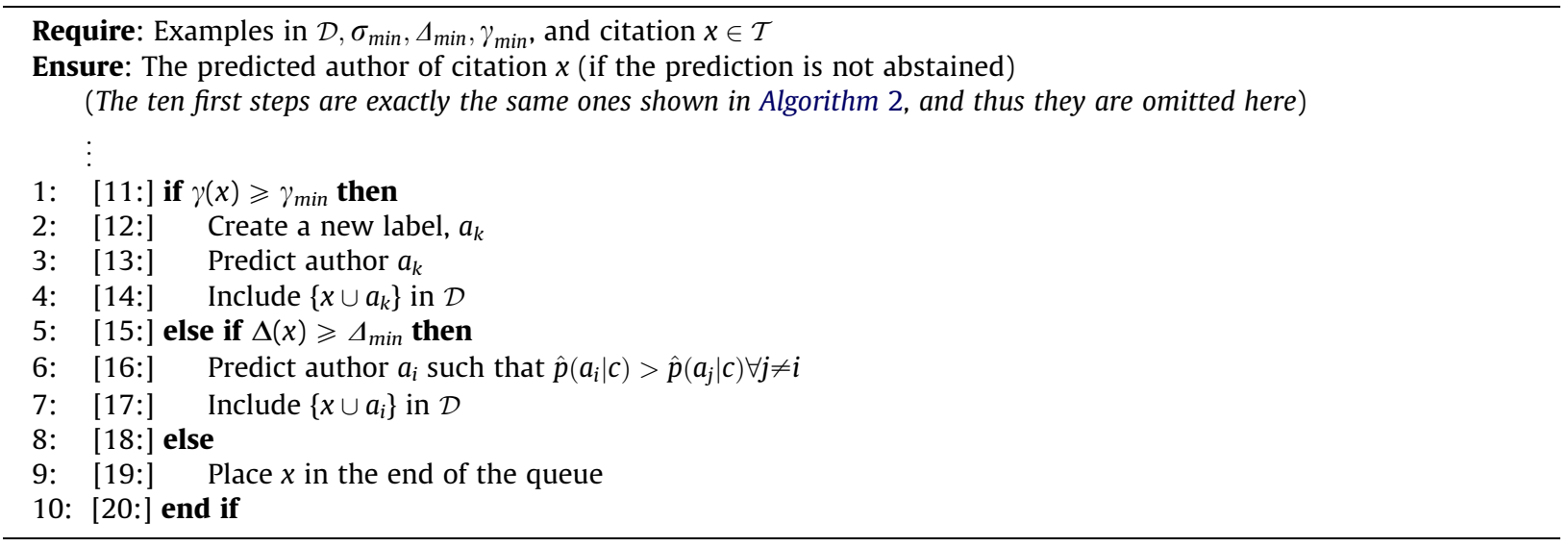




\section{Evaluation}

In this section we present experimental results for the evaluation of the proposed associative disambiguators. We first present the collections employed, evaluation metrics and baselines. Then we discuss the effectiveness of the proposed disambiguators in these collections.

\subsection{Collections}

We used collections of citations extracted from DBLP and from BDBComp. Each citation consists of the title of the work, a list of coauthor names, and the title of the publication venue (these are the most common features present on citations). Preprocessing involved standardizing coauthor names using only the initial letter of the first name along with the full last name, removing punctuation and stopwords of publication and venue titles, stemming publication and venue titles using Porter's algorithm (Porter, 1980), and grouping authors with the same first name initial and the same last name (i.e., creating the ambiguous groups).

Table 3 shows more detailed information about the collections and their ambiguous groups. Disambiguation is particularly difficult in ambiguous groups such as the "C. Chen" group, in which the correct author must be selected from 60 possible authors, and in ambiguous groups such as the "J. Silva" group, in which the majority of authors appears in only one citation. Fig. 1 shows the authorship distribution within each of two representative groups of each collection. Notice that, for a given group, few authors are very prolific and appear in several citations, while most of the authors appear in only few citations (the same trend is observed in all groups of DBLP and BDBComp). This is an intrinsic characteristic of scientific publications, as pointed out in (Liming \& Lihua, 2005).

\subsection{Evaluation Metrics}

Disambiguation effectiveness, that is, the ability to properly select the author of a citation, is assessed through precision, recall and $F_{1}$ metrics. Precision $p$ is defined as the proportion of correctly disambiguated citations (i.e., citations for which the corresponding author was correctly predicted by the disambiguator). Recall $r$ is defined as the proportion of correctly disambiguated citations out of all the citations having the target author. $\mathrm{F}_{1}$ is defined as the harmonic mean of precision and recall (i.e., $\frac{2 p r}{p+r}$ ). Macro- and micro-averaging were applied to $F_{1}$ to get single effectiveness values. For $F_{1}$ macro-averaging $\left(\operatorname{macroF}_{1}\right)$, scores were first computed for individual authors and then averaged over all authors. For $\mathrm{F}_{1}$ micro-averaging $\left(\mathrm{micro}_{1}\right)$, the decisions for all authors were counted in a joint pool. $\mathrm{MacroF}_{1}$ and $\mathrm{microF}_{1}$ are the primary metrics used in this paper.

\subsection{Baselines}

We used the two supervised name disambiguators proposed in (Han et al., 2004) as baselines. The first disambiguator uses the Naive Bayes probability model (Mitchell, 1997) and the second one uses Support Vector Machines (SVM) (Cortes \& Vapnik, 1995). It is worth mentioning that, as described in the literature (Han et al., 2004), these disambiguators are representative supervised disambiguation methods for bibliographic citations that use the same set of features as us (coauthor names, work title and publication venue title) for the disambiguation task. For further details on these methods, please refer to (Han et al., 2004). We also employed the k-way spectral clustering (Han, Zha, et al., 2005) unsupervised disambiguator as baseline (in order to evaluate scenarios where no training example is available).

\subsection{Results}

All experiments were performed on a Linux-based PC with an Intel Core 2 Duo $1.83 \mathrm{GHz}$ processor and 2GBytes RAM. All results presented were found to be statistically significant at the $95 \%$ confidence level when tested with the two-tailed

Table 3

The DBLP and BDBComp collections

\begin{tabular}{|c|c|c|c|c|c|}
\hline \multicolumn{3}{|l|}{ DBLP } & \multicolumn{3}{|l|}{ BDBComp } \\
\hline Ambiguous group & \#Citations & \#Authors & Ambiguous group & \#Citations & \#Authors \\
\hline A. Gupta & 576 & 26 & A. Oliveira & 52 & 16 \\
\hline A. Kumar & 243 & 14 & A. Silva & 64 & 32 \\
\hline C. Chen & 798 & 60 & F. Silva & 26 & 20 \\
\hline D. Johnson & 368 & 15 & J. Oliveira & 48 & 18 \\
\hline J. Martin & 112 & 16 & J. Silva & 36 & 17 \\
\hline J. Robinson & 171 & 12 & J. Souza & 35 & 11 \\
\hline J. Smith & 921 & 29 & L. Silva & 33 & 18 \\
\hline K. Tanaka & 280 & 10 & M. Silva & 21 & 16 \\
\hline M. Brown & 153 & 13 & R. Santos & 20 & 16 \\
\hline M. Jones & 260 & 13 & R. Silva & 28 & 20 \\
\hline M. Miller & 405 & 12 & - & - & - \\
\hline
\end{tabular}



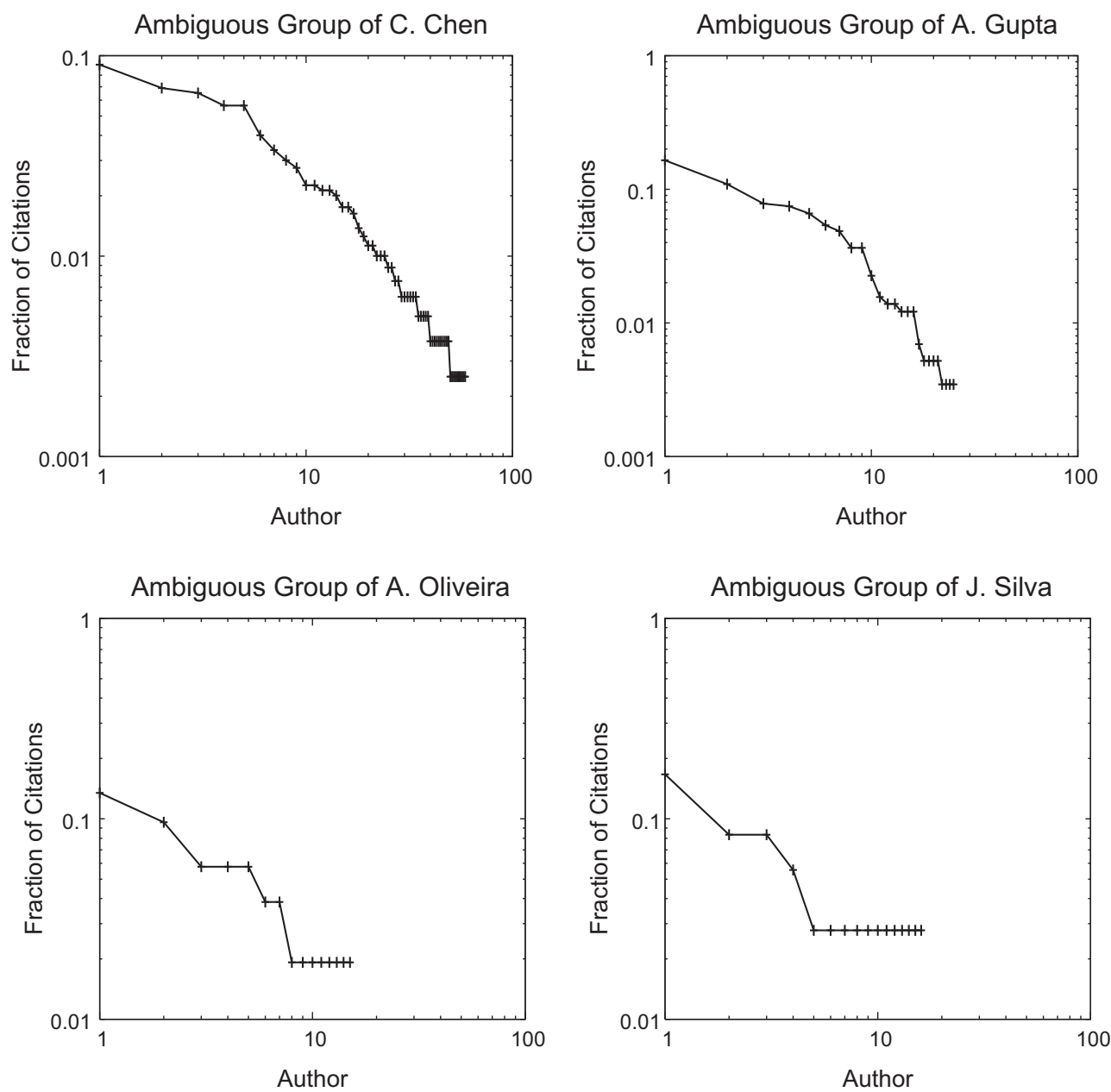

Fig. 1. Authorship distribution within each ambiguous group. Authors ( $x$-axis) are sorted in decreasing order of prolificness (i.e., more prolific authors appear in the first positions).

paired $t$-test. For EAND, LAND and SLAND we set $\sigma_{\min }=0.05$. For SLAND, in particular, we investigated the sensitivity to parameters $\gamma_{\min }$ and $\Delta_{\min }$. RBF kernels were used for SVM and we used a LibSVM tool (Chang \& Lin, 2001) for finding their optimum parameters for each ambiguous group. We used a non-parametric implementation for Naive Bayes (Domingos \& Pazzani, 1997).

How effective are associative disambiguators compared with the baselines?

We evaluate the disambiguation effectiveness obtained by different disambiguators using DBLP and BDBComp collections. Specifically, we performed 10 -fold cross-validation within each ambiguous group, and the final result associated with each group represents the average of the ten runs. Table 4 shows microF $_{1}$ and macroF $_{1}$ values for Naive Bayes, SVM, EAND and LAND in each ambiguous group. ${ }^{5}$ In terms of microF $_{1}$, EAND is in close rivalry with Naive Bayes and SVM, being a little better than Naive Bayes and little worse than SVM. LAND, on the other hand, shows an outstanding effectiveness, being the best performer in all ambiguous groups, with gains ranging from 1.8\% (group of "K. Tanaka") to 15.2\% (group of "J. Martin"), and also on average (with gains of more than $6.3 \%$, compared to Naive Bayes). Disambiguation effectiveness in terms of macroF $\mathrm{F}_{1}$ is also notorious. Again, EAND is very competitive with SVM and Naive Bayes, and LAND is the best performer in all ambiguous groups, with gains ranging from 2.5\% (group of "K. Tanaka") to 23.4\% (group of "D. Johnson"). On average, gains range from $12.1 \%$ (compared to SVM) to $16.9 \%$ (compared to Naive Bayes). Even better results can be observed in the case of the BDBComp collection, as shown in Table 5. In this case, overall gains range from 32\% (group of "A. Oliveira") to 466\% (group of "F. Silva").

The main reason for this impressive disambiguation effectiveness is depicted in Fig. 2. We selected some ambiguous groups, and for each group we sorted the corresponding authors in descending order of popularity ( $x$-axis). Thus, more

\footnotetext{
${ }^{5}$ We considered unfair the inclusion of SLAND in this comparison because it is the only disambiguator that performs self-training, possibly using citations in the test set as additional examples.
} 
Table 4

MicroF $_{1}$ and MacroF $_{1}$ values for the DBLP collection. Best results, including statistical ties, are highlighted in bold.

\begin{tabular}{|c|c|c|c|c|}
\hline Ambiguous group & NB & SVM & EAND & LAND \\
\hline A. Gupta & $0.883 \pm 0.036$ & $0.874 \pm 0.043$ & $0.866 \pm 0.040$ & $\mathbf{0 . 9 2 1} \pm 0.027$ \\
\hline A. Kumar & $0.837 \pm 0.077$ & $0.873 \pm 0.077$ & $\mathbf{0 . 8 9 9} \pm 0.053$ & $\mathbf{0 . 9 2 8} \pm 0.059$ \\
\hline C. Chen & $0.794 \pm 0.037$ & $0.789 \pm 0.039$ & $0.793 \pm 0.038$ & $\mathbf{0 . 8 5 5} \pm 0.037$ \\
\hline D. Johnson & $0.833 \pm 0.078$ & $0.876 \pm 0.068$ & $0.869 \pm 0.050$ & $\mathbf{0 . 9 1 1} \pm 0.036$ \\
\hline J. Martin & $0.719 \pm 0.105$ & $0.748 \pm 0.142$ & $0.747 \pm 0.141$ & $\mathbf{0 . 8 2 7} \pm 0.090$ \\
\hline J. Robinson & $\mathbf{0 . 8 6 1} \pm 0.113$ & $0.869 \pm 0.083$ & $0.825 \pm 0.101$ & $0.934 \pm 0.069$ \\
\hline J. Smith & $0.873 \pm 0.032$ & $0.909 \pm 0.033$ & $0.873 \pm 0.015$ & $\mathbf{0 . 9 2 8} \pm 0.028$ \\
\hline K. Tanaka & $0.917 \pm 0.041$ & $0.936 \pm 0.053$ & $\mathbf{0 . 9 2 8} \pm 0.045$ & $\mathbf{0 . 9 5 3} \pm 0.054$ \\
\hline M. Brown & $0.878 \pm 0.096$ & $\mathbf{0 . 8 7 9} \pm 0.085$ & $\mathbf{0 . 8 8 6} \pm 0.087$ & $\mathbf{0 . 9 3 4} \pm 0.064$ \\
\hline M. Jones & $\mathbf{0 . 8 5 5} \pm 0.064$ & $\mathbf{0 . 8 5 7} \pm 0.051$ & $0.844 \pm 0.087$ & $\mathbf{0 . 8 8 4} \pm 0.052$ \\
\hline M. Miller & $0.935 \pm 0.047$ & $0.926 \pm 0.026$ & $0.931 \pm 0.043$ & $\mathbf{0 . 9 6 0} \pm 0.032$ \\
\hline Average & $0.857 \pm 0.023$ & $0.870 \pm 0.023$ & $0.861 \pm 0.012$ & $\mathbf{0 . 9 1 1} \pm 0.018$ \\
\hline A. Gupta & $0.763 \pm 0.081$ & $0.761 \pm 0.0988$ & $0.739 \pm 0.079$ & $\mathbf{0 . 8 6 6} \pm 0.056$ \\
\hline A. Kumar & $0.715 \pm 0.116$ & $0.749 \pm 0.142$ & $\mathbf{0 . 8 1 0} \pm 0.087$ & $\mathbf{0 . 8 3 7} \pm 0.097$ \\
\hline C. Chen & $0.654 \pm 0.060$ & $0.696 \pm 0.061$ & $0.682 \pm 0.043$ & $0.795 \pm 0.061$ \\
\hline D. Johnson & $0.694 \pm 0.099$ & $0.790 \pm 0.126$ & $0.737 \pm 0.100$ & $\mathbf{0 . 8 5 6} \pm 0.074$ \\
\hline J. Martin & $0.647 \pm 0.115$ & $0.646 \pm 0.123$ & $\mathbf{0 . 6 9 6} \pm 0.145$ & $\mathbf{0 . 7 5 8} \pm 0.122$ \\
\hline J. Robinson & $0.822 \pm 0.135$ & $0.844 \pm 0.092$ & $0.782 \pm 0.127$ & $\mathbf{0 . 9 3 4} \pm 0.068$ \\
\hline J. Smith & $0.672 \pm 0.100$ & $0.742 \pm 0.077$ & $0.685 \pm 0.097$ & $\mathbf{0 . 8 1 8} \pm 0.102$ \\
\hline K. Tanaka & $0.835 \pm 0.089$ & $\mathbf{0 . 8 8 2} \pm 0.105$ & $\mathbf{0 . 8 4 8} \pm 0.086$ & $0.904 \pm 0.111$ \\
\hline M. Brown & $0.843 \pm 0.124$ & $\mathbf{0 . 8 3 4} \pm 0.143$ & $\mathbf{0 . 8 5 8} \pm 0.110$ & $\mathbf{0 . 9 0 9} \pm 0.106$ \\
\hline M. Jones & $0.721 \pm 0.127$ & $0.731 \pm 0.118$ & $0.719 \pm 0.132$ & $0.806 \pm 0.104$ \\
\hline M. Miller & $0.735 \pm 0.156$ & $0.694 \pm 0.117$ & $0.733 \pm 0.155$ & $\mathbf{0 . 8 2 9} \pm 0.134$ \\
\hline Average & $0.712 \pm 0.037$ & $0.743 \pm 0.045$ & $0.730 \pm 0.027$ & $\mathbf{0 . 8 3 3} \pm 0.043$ \\
\hline
\end{tabular}

Table 5

$\mathrm{MicroF}_{1}$ and $\mathrm{MacroF}_{1}$ values for the $\mathrm{BDBComp}$ collection. Best results, including statistical ties, are highlighted in bold.

\begin{tabular}{|c|c|c|c|c|}
\hline Ambiguous group & NB & SVM & EAND & LAND \\
\hline A. Oliveira & $0.497 \pm 0.303$ & $\mathbf{0 . 5 0 0} \pm 0.327$ & $\mathbf{0 . 5 1 5} \pm 0.331$ & $\mathbf{0 . 6 5 7} \pm 0.236$ \\
\hline A. Silva & $0.283 \pm 0.190$ & $0.283 \pm 0.190$ & $0.258 \pm 0.188$ & $\mathbf{0 . 5 2 1} \pm 0.172$ \\
\hline F. Silva & $0.050 \pm 0.158$ & $0.050 \pm 0.158$ & $0.083 \pm 0.166$ & $\mathbf{0 . 2 8 3} \pm 0.352$ \\
\hline J. Oliveira & $0.450 \pm 0.258$ & $0.415 \pm 0.208$ & $\mathbf{0 . 4 1 2} \pm 0.239$ & $\mathbf{0 . 4 5 5} \pm 0.259$ \\
\hline J. Silva & $0.400 \pm 0.214$ & $0.458 \pm 0.249$ & $0.428 \pm 0.244$ & $\mathbf{0 . 6 2 5} \pm 0.267$ \\
\hline J. Souza & $0.617 \pm 0.324$ & $\mathbf{0 . 6 5 0} \pm 0.266$ & $0.622 \pm 0.274$ & $\mathbf{0 . 7 0 8} \pm 0.201$ \\
\hline L. Silva & $0.217 \pm 0.269$ & $0.200 \pm 0.243$ & $0.230 \pm 0.248$ & $\mathbf{0 . 6 0 0} \pm 0.251$ \\
\hline M. Silva & $0.000 \pm 0.000$ & $0.000 \pm 0.000$ & $0.000 \pm 0.000$ & $\mathbf{0 . 2 5 0} \pm 0.264$ \\
\hline R. Santos & $0.000 \pm 0.000$ & $0.000 \pm 0.000$ & $0.000 \pm 0.000$ & $\mathbf{0 . 2 0 0} \pm 0.350$ \\
\hline R. Silva & $0.117 \pm 0.193$ & $0.117 \pm 0.193$ & $0.107 \pm 0.232$ & $\mathbf{0 . 2 6 7} \pm 0.251$ \\
\hline Average & $0.263 \pm 0.113$ & $0.267 \pm 0.230$ & $0.265 \pm 0.238$ & $\mathbf{0 . 4 5 7} \pm 0.192$ \\
\hline A. Oliveira & $0.056 \pm 0.042$ & $\mathbf{0 . 1 2 5} \pm 0.138$ & $\mathbf{0 . 1 1 2} \pm 0.094$ & $\mathbf{0 . 1 7 2} \pm 0.105$ \\
\hline A. Silva & $0.022 \pm 0.021$ & $0.033 \pm 0.033$ & $0.035 \pm 0.029$ & $\mathbf{0 . 0 8 9} \pm 0.047$ \\
\hline F. Silva & $0.003 \pm 0.010$ & $0.025 \pm 0.079$ & $0.055 \pm 0.053$ & $\mathbf{0 . 1 0 5} \pm 0.173$ \\
\hline J. Oliveira & $0.047 \pm 0.025$ & $0.085 \pm 0.064$ & $0.074 \pm 0.022$ & $\mathbf{0 . 1 1 2} \pm 0.115$ \\
\hline J. Silva & $0.048 \pm 0.028$ & $0.133 \pm 0.108$ & $0.089 \pm 0.058$ & $\mathbf{0 . 1 9 5} \pm 0.118$ \\
\hline J. Souza & $0.086 \pm 0.056$ & $0.177 \pm 0.129$ & $0.109 \pm 0.071$ & $\mathbf{0 . 2 4 0} \pm 0.117$ \\
\hline L. Silva & $0.035 \pm 0.031$ & $0.021 \pm 0.024$ & $0.047 \pm 0.031$ & $\mathbf{0 . 1 3 6} \pm 0.095$ \\
\hline M. Silva & $0.000 \pm 0.000$ & $0.000 \pm 0.000$ & $0.000 \pm 0.000$ & $\mathbf{0 . 0 6 3} \pm 0.077$ \\
\hline R. Santos & $0.000 \pm 0.000$ & $0.000 \pm 0.000$ & $0.000 \pm 0.000$ & $\mathbf{0 . 0 5 5} \pm 0.089$ \\
\hline R. Silva & $0.014 \pm 0.022$ & $\mathbf{0 . 0 4 2} \pm 0.104$ & $\mathbf{0 . 0 5 2} \pm 0.089$ & $\mathbf{0 . 0 6 8} \pm 0.101$ \\
\hline Average & $0.031 \pm 0.029$ & $0.064 \pm 0.062$ & $0.057 \pm 0.044$ & $\mathbf{0 . 1 2 4} \pm 0.062$ \\
\hline
\end{tabular}

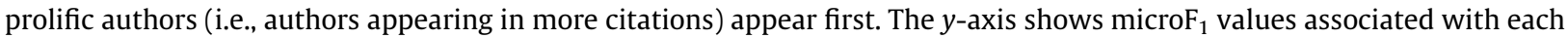
author. In general, more prolific authors are better disambiguated. All disambiguators perform better, in general, when dealing with more popular/prolific authors. Disambiguation effectiveness tends to decrease with prolificness and this is mainly due to the reduction in the amount of examples available during training (i.e., there is only few citations associated with these authors). The crucial point is that, as shown in Fig. 2, LAND is able to focus on producing functions that are suitable to disambiguate specific citations. This is because LAND builds the disambiguation function on a demand-driven basis, achieving higher effectiveness in less prolific authors, since important evidence supporting such authors are not discarded 

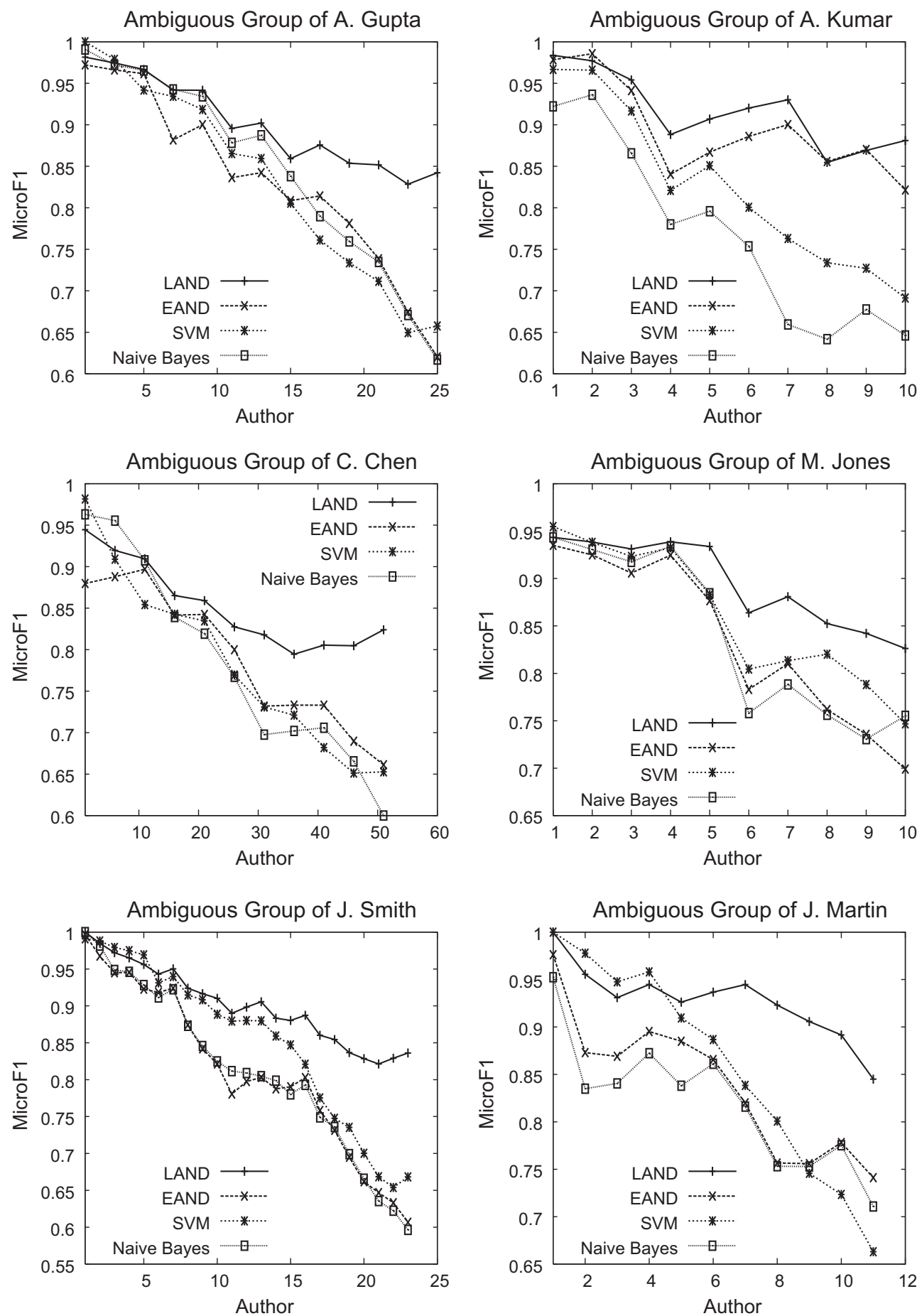

Fig. 2. Relationship between microF $_{1}$ and author prolificness. Authors ( $x$-axis) are sorted in decreasing order of prolificness (i.e., more prolific authors appear in the first positions).

during rule extraction (i.e., multiple cut-off values are applied). Since less prolific authors, when considered together, correspond to a large number of citations, the ability of generating specific disambiguation functions according to a particular citation incurs in impressive gains in effectiveness. 
How the different disambiguators perform with limited labeling efforts?

We evaluate the disambiguation effectiveness obtained by each disambiguator by varying the fraction of available examples. None of the disambiguators evaluated in this experiment exploit unlabeled data (which, in this case, correspond to citations in the test set) to increase the number of available examples (note that LAND simply uses citations in the test set to guide the lazy search for useful rules, but not as additional training examples). For this experiment, we performed 5-fold cross-validation within each ambiguous group and, from the original training data associated with each fold, we produce 10 subsets, where each subset contains a different fraction of examples which were randomly selected from the training data (i.e., $10 \%, 20 \%, \ldots, 100 \%$ of the citations in the training data associated with each fold). In the results, which are depicted in Fig. 3, each point represents the average of the five runs, which are then averaged over all ambiguous groups (i.e., similar to the last line of Table 4).

For both collections, the effectiveness of all disambiguators are very similar when only few examples are available. However, when more examples are available, LAND achieves superior effectiveness compared to the baselines. In such cases, for the DBLP collection, LAND showed a significant improvement (both in terms of microF $F_{1}$ and macroF $_{1}$ ). On the other hand, for the BDBComp collection, the effectiveness of Naive Bayes, EAND and LAND are very close. While high effectiveness was observed in the DBLP collection, a very low effectiveness (specially in terms of macroF ${ }_{1}$ ) was obtained in the BDBComp collection. This is because the BDBComp collection contains many authors that appear only in the test set (most of them appearing in only one citation) and, thus, the predictions for the citations being authored by these authors are always wrong (since there is no example supporting these authors in the training data). Next, we will evaluate SLAND, which has the ability to detect unseen authors (i.e., authors appearing only on citations in the test set) and to enhance the training data by incorporating additional examples.

How does $\gamma_{\min }$ impact the effectiveness of SLAND?

We evaluate the effectiveness of SLAND in detecting unseen authors using the BDBComp collection. Differently from the DBLP collection, the BDBComp collection contains many unseen authors. Specifically, $43.5 \%$ of the authors appear only in the test set. For this experiment, we, again, perform 5-fold cross-validation, following the same strategy used in the previous experiment. However, for each fraction of training examples, we varied $\gamma_{\min }$ from 1 to 6 . The results are shown in Fig. 4.
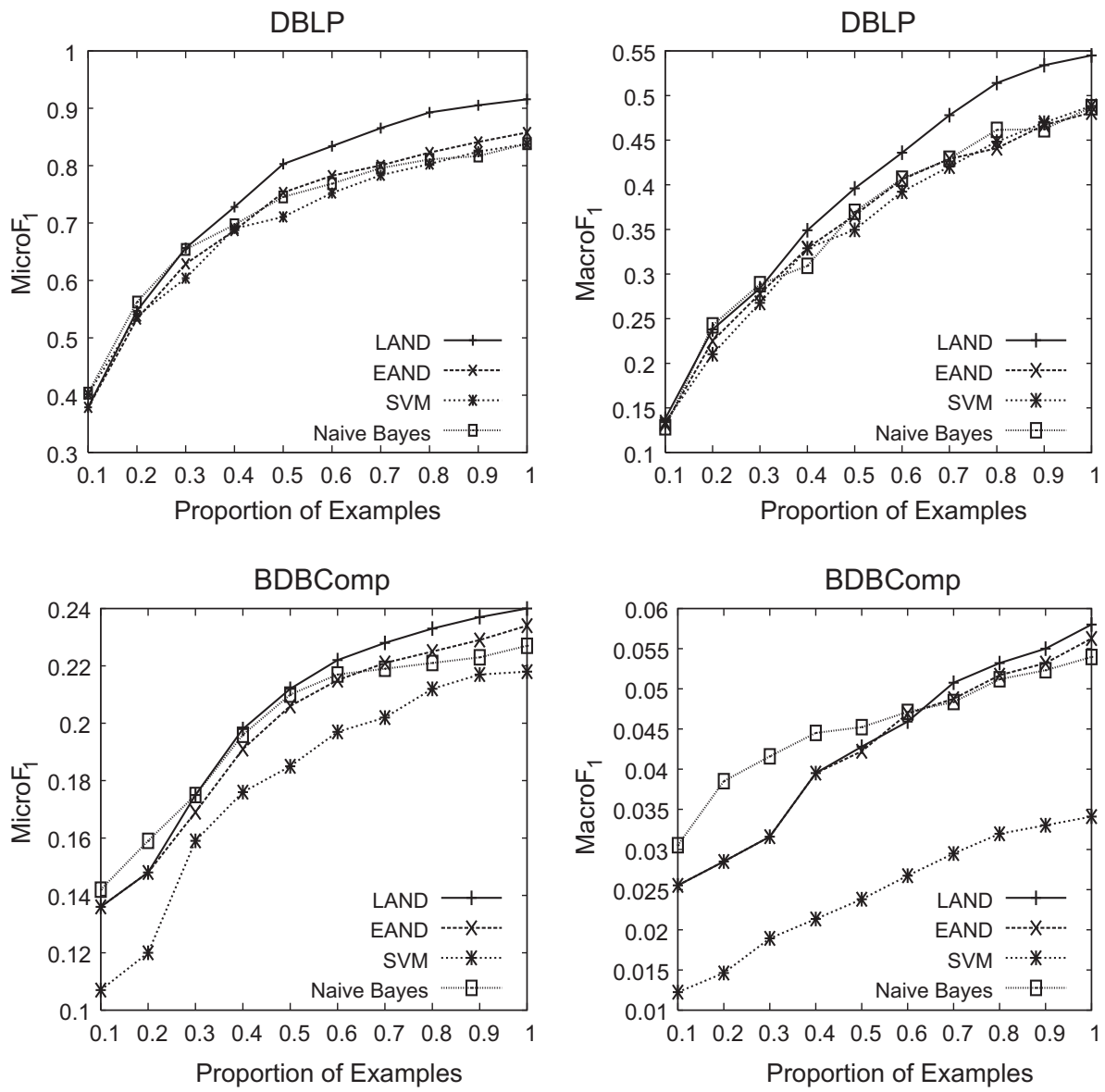

Fig. 3. MicroF $_{1}$ and macroF $_{1}$ values for varying number of examples. 
For the BDBComp collection, the fraction of unseen authors that are detected (number of detected unseen authors divided by total number of unseen authors) increases with $\gamma_{\text {min }}$. This is expected, since the amount of evidence that is required to recognize an author as already seen, increases for higher values of $\gamma_{\min }$. Further, it becomes more difficult to detect an unseen author when the fraction of training examples increases. This is because, in such cases, (1) more authors are seen (i.e., there are more examples), and (2) there is an increase in the amount of available evidence supporting already seen authors.

How does $\Delta_{\text {min }}$ impact the effectiveness of SLAND?

We evaluate the effectiveness of SLAND in incorporating new training examples using the DBLP collection, since this collection contains much more citations in the test set. Again, we perform 5-fold cross-validation, following the same strategy used in the previous experiment. However, for each fraction of training examples, we varied $\Delta_{\min }$ from 0.5 to 0.9 . The results are shown in Fig. 5 . As it can be seen, the effectiveness of SLAND decreases when $\Delta_{\min }$ (i.e., the minimum reliability required to consider a prediction as reliable) is set too high (i.e., $\Delta_{\min }>0.75$ ). Further, the effectiveness also decreases when $\Delta_{\min }$ is set too low (i.e., $\Delta_{\min }<0.65$ ). On one hand, when lower values of $\Delta_{\min }$ are applied, several citations in the test set, which are associated with wrong predictions, are included in the training data (note that the reliability of a prediction decreases with $\Delta_{\min }$ ), hurting effectiveness. On the other hand, when higher values of $\Delta_{\min }$ are applied, only few citations in the test set are included in the training data. For the DBLP collection, SLAND achieves the best effectiveness when $\Delta_{\text {min }}$ is between 0.65 and 0.75 (specially when few training examples are available).

How effective is SLAND compared with LAND?

We now evaluate how the abilities of SLAND improve its effectiveness when compared to LAND. We, again, perform 5fold cross-validation, following the same strategy used in the previous experiments. Fig. 6 shows some of the results. The value associated with each point in each graph is obtained by applying a different combination of $\gamma_{\min }$ and $\Delta_{\min }$, for different fractions of training examples. For the DBLP collection, gains ranging from $18.4 \%$ to $53.8 \%$ are observed when few training examples are available. The improvement decreases when more examples are available, since in this case (1) more authors are seen and (2) additional examples that are included in the training data do not impact so much the final effectiveness. Interestingly, SLAND achieves good effectiveness even when not a single example is available for training. This is possible because, in this case, citations authored by unseen authors are included in the training data, and used as training examples. These gains highlight the advantages of self-training.

Improvements obtained using the BDBComp collection are more impressive. As discussed before, this collection contains several authors that appear in only one citation. LAND (and other completely supervised methods) is not useful in such scenarios, since it is not able to produce correct disambiguation functions for such citations (i.e., if this citation appears only in the test set, then the training data contains no evidence supporting the correct author). SLAND, on the other hand, is highly effective in such cases, being able to detect unseen authors, and to make use of this information to enhance the training data with additional examples. As a result, improvements provided by SLAND range from 241.6\% to 407.1\%. Thus, SLAND is not only able to reduce labeling efforts (as shown in the experiments with the DBLP collection), but it is also able to detect novel and important information (i.e., unseen authors), being highly practical and effective in a variety of scenarios.

How effective is SLAND compared to an unsupervised disambiguator?

We used the DBLP collection to perform a comparison between SLAND $\left(\gamma_{\min }=4, \Delta_{\min }=0.7\right)$, and the k-way spectral clustering disambiguator (Han, Zha, et al., 2005), when no training example is available for any of the disambiguators. We adopted the evaluation methodology proposed in (Han, Zha, et al., 2005), so that we can directly compare the effectiveness

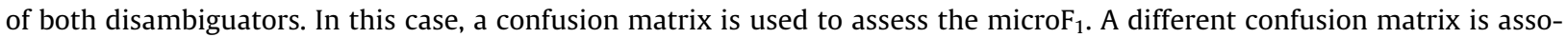
ciated with each ambiguous group, and the final effectiveness is represented by the accuracy averaged over all groups.

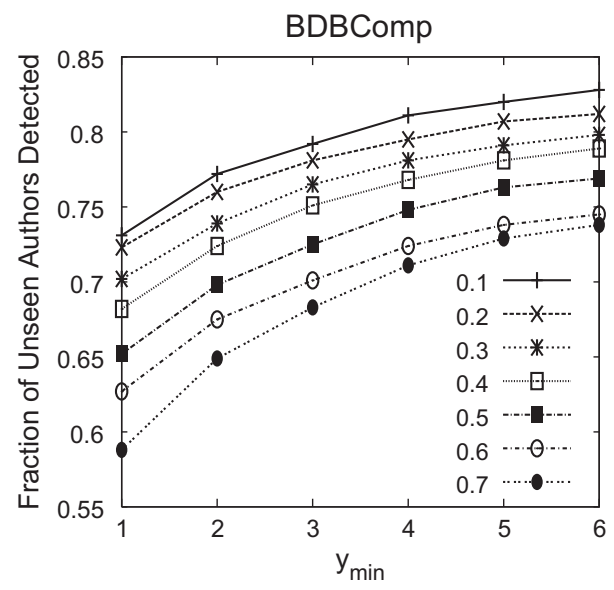

Fig. 4. Sensitivity to $\gamma_{\min }$ (each curve is associated with a different fraction of training examples). 


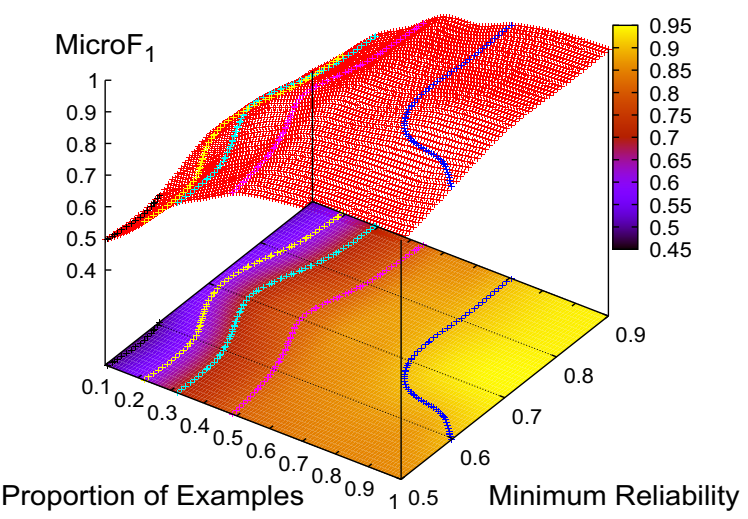

Fig. 5. Sensitivity to $\Delta_{\min }$.
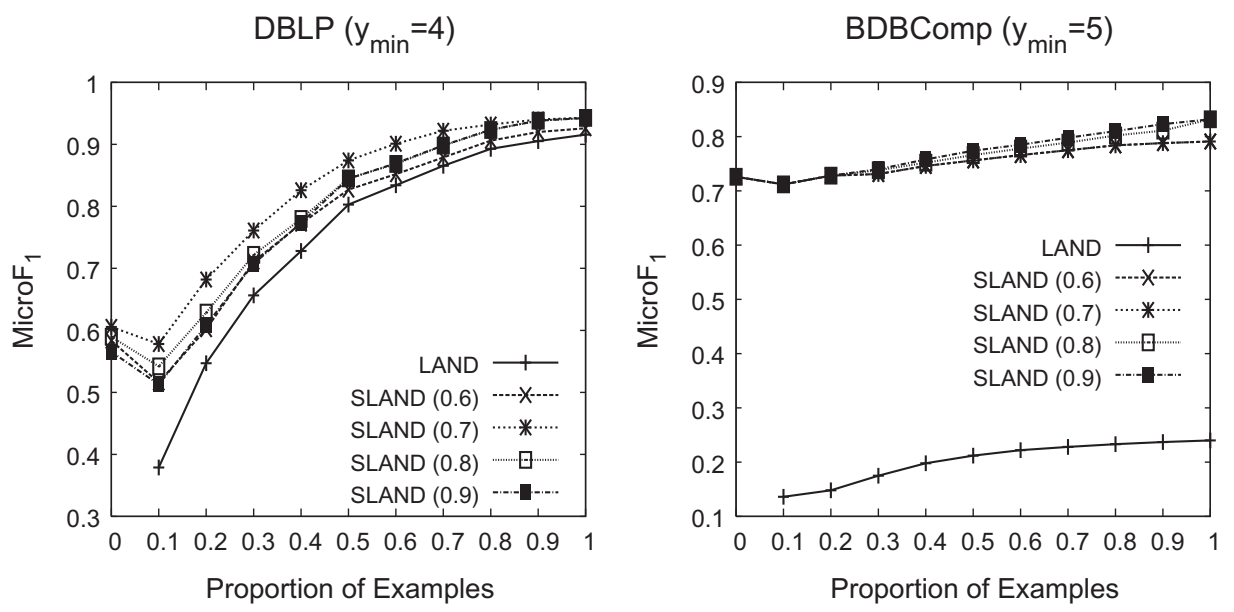

Fig. 6. MicroF $F_{1}$ values for different $\Delta_{\min }$ and $\gamma_{\min }\left(\Delta_{\min }\right.$ values are between parentheses).

Both disambiguators are statistically tied on almost all ambiguous groups (see Table 6). The K-way spectral clustering disambiguator obtained superior effectiveness on three ambiguous groups, while SLAND was superior in one ambiguous group. It is important to notice that the k-way spectral clustering disambiguator takes as input the correct number of clusters to be generated, that is, if there are $m$ authors in a group, then this group is clustered into exactly $m$ clusters (Han, Zha, et al., 2005). This is clearly unrealistic in an actual or practical scenario, but provides something closer to an upper-bound for an

Table 6

SLAND compared to the K-way spectral clustering disambiguator in terms of microF $F_{1}$ on the DBLP collection. Best results, including statistical ties, are highlighted in bold.

\begin{tabular}{lll}
\hline Ambiguous group & \multicolumn{2}{l}{ MicroF 1} \\
\cline { 2 - 3 } & SLAND & K-Way SC \\
\hline A. Gupta & $0.453 \pm 0.050$ & $\mathbf{0 . 5 4 6} \pm 0.048$ \\
A. Kumar & $\mathbf{0 . 5 5 5} \pm 0.150$ & $\mathbf{0 . 5 0 5} \pm 0.029$ \\
C. Chen & $0.365 \pm 0.052$ & $\mathbf{0 . 6 0 7} \pm 0.050$ \\
D. Johnson & $\mathbf{0 . 7 1 0} \pm 0.062$ & $0.561 \pm 0.081$ \\
J. Martin & $0.786 \pm 0.058$ & $\mathbf{0 . 9 3 9} \pm 0.062$ \\
J. Robinson & $\mathbf{0 . 6 6 2} \pm 0.103$ & $\mathbf{0 . 6 9 3} \pm 0.051$ \\
J. Smith & $\mathbf{0 . 4 4 4} \pm 0.057$ & $\mathbf{0 . 5 0 0} \pm 0.097$ \\
K. Tanaka & $\mathbf{0 . 5 5 4} \pm 0.099$ & $\mathbf{0 . 6 2 6} \pm 0.120$ \\
M. Brown & $\mathbf{0 . 6 8 0} \pm 0.133$ & $\mathbf{0 . 7 5 9} \pm 0.143$ \\
M. Jones & $\mathbf{0 . 5 0 4} \pm 0.179$ & $\mathbf{0 . 6 2 8} \pm 0.083$ \\
M. Miller & $\mathbf{0 . 6 9 9} \pm 0.126$ & $\mathbf{0 . 4 7 9} \pm 0.117$ \\
Average & $\mathbf{0 . 5 8 3} \pm 0.097$ & $\mathbf{0 . 6 2 2} \pm 0.080$ \\
\hline
\end{tabular}


unsupervised disambiguator that has privileged information. SLAND, on the other hand, does not use this information, and works by detecting unseen authors, and incrementally adding new examples to the training data. Other point worth mentioning is that, as shown in Fig. 6, with small labeling efforts, the effectiveness of SLAND can be much improved (greatly outperfoming the unsupervised disambiguator), demonstrating that SLAND is very cost-effective.

\section{Conclusions and future work}

Name disambiguation, in the context of bibliographic citations, is the problem of determining whether records in a collection of publications refer to the same person. This problem is widespread in many large-scale digital libraries, such as Citeseer, Google Scholar and DBLP.

Authorship frequency follows a very skewed distribution. Few authors are very prolific while most of the authors are included in only few citations. This property seems to affect the effectiveness of disambiguators based on machine learning techniques such as Naive Bayes and SVM. Thus, in this article we propose a novel approach for name disambiguation that uncovers associations between bibliographic features and authors. The proposed disambiguators based on this approach were evaluated showing competitive results. LAND, in particular, which is based on a demand-driven rule generation process, showed superior effectiveness when compared to the state-of-the-art. A deep analysis revealed that the outstanding effectiveness of LAND is mainly because it builds disambiguation functions on a demand-driven basis, so that authors appearing in only few citations can be better disambiguated. Other factors that greatly affect disambiguation effectiveness include the prohibitive cost of labeling vast amounts of examples and the appearance of unseen authors. Thus, we extend LAND with the self-training ability. The resulting disambiguator, SLAND, drastically reduces the amount of examples required to build effective disambiguation functions, and is also competent in detecting unseen authors. The self-training ability makes SLAND highly effective and practical. In fact, we already have initial evidence that SLAND can be very effective even in situations in which the training data is automatically produced, i.e., with no manual labeling at all (Ferreira, Veloso, Gonçalves, \& Laender, 2010).

As future work, we intend to perform experiments with other collections, particularly from fields other than Computer Science, as well as considering other features like those extracted from headers of scientific papers (e.g., affiliation, address, e-mail), obtained from collaborative social networks, or from the topics or categories of the citations.

\section{Acknowledgments}

This research is partially funded by the National Institute of Science and Technology for the Web (InWeb) (MCT/CNPq/ FAPEMIG Grant No. 573871/2008-6), and by the authors's individual research Grants from CAPES, CNPq, and FAPEMIG.

\section{References}

Agrawal, R., Imielinski, T., \& Swami, A. (1993). Mining association rules between sets of items in large databases. In Proceedings of the 1993 ACM SIGMOD international conference on management of data (pp. 207-216). Washington, USA.

Bekkerman, R., \& McCallum, A. (2005). Disambiguating web appearances of people in a social network. In Proceedings of the 14th international conference on world wide web (pp. 463-470). Chiba, Japan.

Bhattacharya, I., \& Getoor, L. (2006). A latent dirichlet model for unsupervised entity resolution. In Proceedings of the Sixth SIAM international conference on data mining. Bethesda, MD, USA.

Bhattacharya, I., \& Getoor, L. (2007). Collective entity resolution in relational data. ACM Transactions on Knowledge Discovery from Data, 1.

Chang, C. -C., \& Lin, C. -J. (2001). LibSVM: A library for support vector machines. Software available at <http://www.csie.ntu.edu.tw/ cjlin/libsvm>.

Cortes, C., \& Vapnik, V. (1995). Support-vector networks. Machine Learning, 20, 273-297.

Cota, R. G., Ferreira, A. A., Nascimento, C., Gonçalves, M. A., \& Laender, A. H. F. (2010). An unsupervised heuristic-based hierarchical method for name disambiguation in bibliographic citations. JASIST, 61, 1853-1870.

Cota, R. G., Gonçalves, M. A., \& Laender, A. H. F. (2007). A heuristic-based hierarchical clustering method for author name disambiguation in digital libraries. In Proceedings of the XXII Brazilian symposium on databases (pp. 20-34). João Pessoa, Paraiba, Brazil.

Culotta, A., Kanani, P., Hall, R., Wick, M., \& McCallum, A. (2007). Author disambiguation using error-driven machine learning with a ranking loss function. In International workshop on information integration on the web. Vancouver, Canada.

Dhillon, I. S., Guan, Y., \& Kulis, B. (2005). A fast kernel-based multilevel algorithm for graph clustering. In Proceedings of the 11th ACM SIGKDD international conference on knowledge discovery and data mining (pp. 629-634). Chicago, Illinois, USA.

Domingos, P., \& Pazzani, M. (1997). On the optimality of the simple Bayesian classifier under zero-one loss. Machine Learning, 29, 103-137.

Ester, M., Kriegel, H. -P., Sander, J., \& Xu, X. (1996). A density-based algorithm for discovering clusters in large spatial databases with noise. In Proceedings of the 2nd international conference on knowledge discovery and data mining (pp. 226-231). Portland, Oregon.

Ferreira, A. A., Veloso, A., Gonçalves, M. A., \& Laender, A. H. F. (2010). Effective self-training author name disambiguation in scholarly digital libraries. In Proceedings of the 2010 ACM/IEEE joint conference on digital libraries (pp. 39-48). Gold Coast, Queensland, Australia.

Goethals, B., \& Zaki, M. (2004). Advances in frequent itemset mining implementations: report on FIMI'03. SIGKDD Explorations, 6, $109-117$.

Han, H., Giles, C. L., Zha, H., Li, C., \& Tsioutsiouliklis, K. (2004). Two supervised learning approaches for name disambiguation in author citations. In Proceedings of the 4th ACM/IEEE-CS joint conference on digital libraries (pp. 296-305). Tuscon, USA.

Han, H., Xu, W., Zha, H., \& Giles, C. L. (2005). A hierarchical naive Bayes mixture model for name disambiguation in author citations. In Proceedings of the 2005 ACM symposium on applied computing (pp. 1065-1069). Santa Fe, New Mexico, USA.

Han, H., Zha, H., \& Giles, C. L. (2005). Name disambiguation in author citations using a k-way spectral clustering method. In Proceedings of the 5th ACM/IEEE joint conference on digital libraries (pp. 334-343). Denver, CO, USA.

Huang, J., Ertekin, S., \& Giles, C. L. (2006). Efficient name disambiguation for large-scale databases. In Proceedings of the 10th European conference on principles and practice of knowledge discovery in databases (pp. 536-544). Berlin, Germany.

Kanani, P., McCallum, A., \& Pal, C. (2007). Improving author coreference by resource-bounded information gathering from the web. In Proceedings of the 20th international joint conference on artificial intelligence (pp. 429-434). Hyderabad, India. 
Kang, I.-S., Na, S.-H., Lee, S., Jung, H., Kim, P., Sung, W.-K., et al (2009). On co-authorship for author disambiguation. Information Processing E Management, 45, 84-97.

Lee, D., Kang, J., Mitra, P., Giles, C. L., \& On, B.-W. (2007). Are your citations clean? Communications of the ACM, 50, 33-38.

Liming, L., \& Lihua, L. (2005). Scientific publication activities of 32 countries. Scientometrics, 26, 263-273.

Malin, B. (2005). Unsupervised name disambiguation via social network similarity. In Proceedings of the workshop on link analysis, counterterrorism, and security, at the SIAM international conference on data mining (pp. 93-102). Newport Beach, CA.

Mitchell, T. M. (1997). Machine learning. New York, NY, USA: McGraw-Hill.

On, B. -W., Elmacioglu, E., Lee, D., Kang, J., \& Pei, J. (2006). An effective approach to entity resolution problem using quasi-clique and its application to digital libraries. In Proceedings of the 6th ACM/IEEE-CS joint conference on digital libraries (pp. 51-52). Chapel Hill, NC, USA.

On, B. -W., \& Lee, D. (2007). Scalable name disambiguation using multi-level graph partition. In Proceedings of the 7th SIAM international conference on data mining (pp. 575-580). Minneapolis, Minnesota, USA.

On, B. -W., Lee, D., Kang, J., \& Mitra, P. (2005). Comparative study of name disambiguation problem using a scalable blocking-based framework. In Proceedings of the 5th ACM/IEEE joint conference on digital libraries (pp. 344-353). Denver, CO, USA.

Pereira, D. A., Ribeiro-Neto, B. A., Ziviani, N., Laender, A. H. F., Gonçalves, M. A., \& Ferreira, A. A. (2009). Using web information for author name disambiguation. In Proceedings of the 2009 ACM/IEEE joint conference on digital libraries (pp. 49-58). Austin, TX, USA.

Porter, M. F. (1980). An algorithm for suffix stripping. Program, 14, 130-137.

Song, Y., Huang, J., Councill, I. G., Li, J., \& Giles, C. L. (2007). Efficient topic-based unsupervised name disambiguation. In Proceedings of the 7th ACM/IEEE joint conference on digital libraries (pp. 342-351). Vancouver, BC, Canada.

Torvik, V. I., Weeber, M., Swanson, D. R., \& Smalheiser, N. R. (2005). A probabilistic similarity metric for medline records: A model for author name disambiguation. Journal of the American Society for Information Science and Technology, 56, 140-158.

Torvik, V. I., \& Smalheiser, N. R. (2009). Author name disambiguation in medline. ACM Transactions on Knowledge Discovery from Data, 3, 1-29.

Treeratpituk, P., \& Giles, C.L. (2009). Disambiguating authors in academic publications using random forests. In Proceedings of the 2009 ACM/IEEE joint conference on digital libraries (pp. 39-48). Austin, TX, USA.

Veloso, A., Meira Jr., W., Cristo, M., Gonçalves, M., \& Zaki, M. (2006). Multi-evidence, multi-criteria, lazy associative document classification. In Proceedings of the 2006 ACM CIKM international conference on information and knowledge management (pp. 218-227). Arlington, USA.

Veloso, A., Meira Jr., W., \& Zaki, M. J. (2006). Lazy associative classification. In Proceedings of the 6th IEEE international conference on data mining (pp. 645654). Hong Kong, China.

Veloso, A., Mosrri, H., Gonçalves, M., \& Meira Jr., W. (2008). Learning to rank at query-time using association rules. In Proceedings of the 31st annual international ACM SIGIR conference on research and development in information retrieval (pp. 267-274). Singapore.

Vu, Q. M., Masada, T., Takasu, A., \& Adachi, J. (2007). Using a knowledge base to disambiguate personal name in web search results. In Proceedings of the 2007 ACM symposium on applied computing (pp. 839-843). Seoul, Korea. 